\title{
Etiologic Link Between Chronic Pancreatitis and Pancreatic Cancer; A Unicentric Experience of Pancreatic Disease Institute of Wuhan Union Hospital of China
}

\author{
Soriba Naby Camara ${ }^{1}$, Sonam Ramdany ${ }^{1}$, Sadamoudou Traore ${ }^{2}$, Aissatou Taran Diallo ${ }^{3}$, Yin Tao ${ }^{1}$, \\ Yang Min ${ }^{1}$, Qin $\mathrm{Qi}^{1}$, Xiang $\mathrm{Li}^{1}$, Jing Yuan Chen ${ }^{1}$, Yong Feng $\mathrm{Li}^{1}$, Biro Diallo ${ }^{4}$, \\ Ahmed Boubacar Barry ${ }^{4}$, Oumar Taibata Balde ${ }^{4}$, Gang Zhao', Cui Jing ${ }^{1}$, Aboubacar Toure ${ }^{3}$, \\ Ibrahima Sory Souare ${ }^{5}$, Naby Daouda Camara ${ }^{3}$, He-shui Wu ${ }^{1}$, Chunyou Wang ${ }^{1,}$, \\ ${ }^{1}$ Department of General Surgery, Pancreatic Center, Union Hospital, Tongji Medical College, Huazhong University of Science and \\ Technology, Wuhan, China \\ ${ }^{2}$ Department of Medical Imaging, Good Shepherd Medical Center Longview, Texas, USA \\ ${ }^{3}$ Department of General Surgery, National Hospital of Ignace Denn, University of Conakry, Conakry, Guinea \\ ${ }^{4}$ Department of Visceral Surgery National Hospital of Donka, University of Conakry, Conakry, Guinea \\ ${ }^{5}$ Department of Neurosurgery, Friendship Hospital Sino Guinea of Kipe, University of Conakry, Conakry, Guinea
}

Email address:

csoribanaby@yahoo.com (Soriba Naby Camara), chunyouwang52@126.com (Chunyou Wang)

\section{To cite this article:}

Soriba Naby Camara, Sonam Ramdany, Sadamoudou Traore, Aissatou Taran Diallo, Yin Tao, Yang Min, Qin Qi, Xiang Li, Jing Yuan Chen, Yong Feng Li, Biro Diallo, Ahmed Boubacar Barry, Oumar Taibata Balde, Gang Zhao, Cui Jing, Aboubacar Toure, IbrahimaSory Souare, NabyDaouda Camara, He-shui Wu, Chunyou Wang. Etiologic Link Between Chronic Pancreatitis and Pancreatic Cancer; A Unicentric Experience of Pancreatic Disease Institute of Wuhan Union Hospital of China. Journal of Surgery. Vol. 3, No. 6, 2015, pp. 59-65. doi: $10.11648 /$ j.js.20150306.12

\begin{abstract}
The aim of this study is to reveal the etiologic link that exists between pancreatic cancer and chronic pancreatitis through our unicentric experience. The clinical and cyto-pathological results of 300 patients were studied prospectively from January to December 2014 in the pancreatic center of Wuhan union hospital in china. Of the 300 pancreatic lesions patients, 196 were male and 104 female with a sex ratio of 1.8:1 $(\mathrm{p}=0.001)$. The factors assessed for the determination of etiologic link were the age, gender, the diameter of the lesion, the antecedents of disease (such as ulcer,cholecystectomy, smoking ,alcohol abuse ), the pancreatic lesion site, the diabetic link, the clinical presentations, the level of transaminases (GGT, ALP), peripheral blood analysis and cytopathological results of both PC and CP groups. According to the clinical data along with the respective results of the examinations, causal factors that link CP and PC are illustrated and it opens the pathway for further detailed studies as an investment for the future.
\end{abstract}

Keywords: Pancreatic Cancer, Chronic Pancreatitis, Etiologic Link, Unicentric Experience, Pancreatic Lesions

\section{Introduction}

Pancreatic cancer (PC) and chronic pancreatitis (CP) are the second and third cause of hospitalization in the pancreatic surgery department of Wuhan Union Hospital of China [1]. PC is a devastating disease and fourth, fifth and seventh leading cause of cancer - related death in the United States, European Union and China, respectively [2]. CP is defined as an inflammatory disease which is characterized by an irreversible conversion of pancreatic parenchyma to fibrous tissue [3]. In the Western world, its incidence has been estimated to be $10 / 100,000$ pa with rising morbidity among females. In $20-30 \%$ of cases of chronic pancreatitis and mass lesions in the pancreas may occur [3]. Several studies have demonstrated a strong link between inflammation and cancer [3]. Virchow was the first to ascertain this link in the 19th century. His hypothesis was thereafter endorsed by several epidemiological researches which acknowledged the etiologic link between CP and PC [4].

Highlighting the causal relationship between CP and PC is necessary since $\mathrm{PC}$ is a disease that has very poor 
prognosis. The diagnosis of PC at an early stage is rare and the by the time it's discovered, it becomes resistance to therapies. Moreover, resection of tumor may not be possible in greater than $80 \%$ of PC patients due to metastasis, leading to a low five- year survival rate [5]. Furthermore, among the risk factors of PC such as obesity, alcohol, cigarettes smoking, diabetes, chronic pancreatitis and age, $\mathrm{CP}$ is the most prominent one [7]. Inflammation to become tumorous need an extended length of period thus inflammatory mediators has a great influence. These inflammatory mediators may cause change in gene expression which may induce activation of neoplastic pathways leading to cancer.

Moreover, during inflammatory response, an increase level of IL-1 (interleukins 1 receptor) in the brain and may cause breakdown of blood brain barrier. Furthermore IL-1 polymorphisms have been found to contribute to genetic susceptibility to some cancers. According to the literature, there are two distinct IL-1 receptors, IL-1R type 1 and IL-1R type II.

Type 1 receptor is primarily responsible for transmitting the inflammatory effects of interleukin-1 while the type 2 receptors act as a suppressor of IL-1 activity by competing for IL-1 binding. Thus IL-1 receptor represent an important counter-regulatory component of the inflammatory response.

In future studies gene array will demonstrate a prospective marker in relation to etiologic link. This article highlights the causal relationship between the two diseases with various factors and the potential markers of pancreatic lesions.

\section{Materials and Methods}

\subsection{Patients}

In Wuhan's pancreatic center of Union hospital, from January to December 2014,300 patients were treated and included in our study. Among them, 266 cases were PC and 34 were CP. The sex ratio was 1.8: $1(\mathrm{P}=0.001)$ with 196 male and 104 male.

All the included cases in our study had done their routine blood examination, the transaminases test, tumors marker test and $\mathrm{CT}$ and MRI.

Patients with small-cell carcinoma, mucinous cystadenocarcinoma, islet cell, papillary cystic neoplasm, or no differentiates adenocarcinoma and biliary diseases were not eligible.

\subsection{Assessment of Transaminases}

GGT in serum was measured using the Szasz method [8], with $\gamma$-glutamyl-p-nitroanilideas substrate and glycylglycine as receptor. ALP activity was determined using the SMA $12 / 60$ auto analyzer with p-Nitro phenyl phosphate. AST and ALT are assayed by the use of SMA 12/60 auto analyzer ultraviolet method. GGT was assayed within $48-60 \mathrm{~h}$ after the serum sample was collected, and ALP, AST, ALT within $24 \mathrm{~h}$. However if they were not examined within 3 hrs.of collection, they were stored at $4^{\circ} \mathrm{C}[9]$.

\subsection{Cytopathological Assessments}

All cyto-pathological samples were processed as cell blocks and were interpreted by the same experienced cytopathologist. The specimens were considered satisfactory in the presence of several non-hemorrhagic small tissue filaments or even tissue core samples. The number of tissue needed to obtain a satisfactory specimen was documented. Briefly, once resected, the material was fixed in buffered formalin, underwent centrifugation, and was immersed in the liquid agarose. Once solidified, the agar cone with the cells in the top was embedded in paraffin to be handled as a routine tissue block. On reviewing the slides, cellularity, presence of loosely cohesive aggregates or single tumor or chronic cells, quality and quantity of cytoplasm, nuclear pleomorphism, chromatin patterns, nucleus to cytoplasm ratio and necrosis were systematically analyzed.

\subsection{Clinical Assessment}

The clinical data were collected using pre-established cards investigation. The following observations were made to highlight the etiologic bonds between the PC and CP:

1. Pain

2. Jaundice

3. Weight loss

4. Diameter of the masses

5. Antecedents of disease (Ulcer, cholecystectomy, smoking, and alcohol abuse)

During operations the macroscopic aspect of spleen was investigated and the peripheral blood, transaminases and tumor markers were analyzed before surgery.

\subsection{Statistical Analysis}

The statistical analysis was carried out using software SPSS version 21, and the figures using the software graph pad. Prism.v5.01. $\mathrm{P}$ value inferior at 0, 05 was regards as statistically significant.

\section{Results}

The activity of the main transaminase among patient in chronic pancreatitis and pancreatic cancer. In pancreatic cancer, the GGT value ranged from 182 to $263 \mathrm{IU} / \mathrm{L}$ with a median value of $223 \mathrm{IU} / \mathrm{L}$ and the ALP ranged from 163 to $208 \mathrm{IU} / \mathrm{L}$ with a mean value of $186 \mathrm{IU} / \mathrm{L}$.

In chronic pancreatitis, the GGT value ranged from 18 to $261 \mathrm{IU} / \mathrm{L}$ with a mean value of $140 \mathrm{IU} / \mathrm{L}$ and ALP value ranged from 70 to $248 \mathrm{IU} / \mathrm{L}$ with a mean value of $159 \mathrm{IU} / \mathrm{L}$.

\section{Discussion}

\subsection{From Inflammation to Cancer}

Inflammation is the primary match that sets the fire of the robust stroma reaction surrounding PC cells. PC mass constitute of a stroma composed of fibroblasts, vessels and leukocytes. Leukocytes of the inflammatory cells are the 
major actors engaged in initiating and sustaining the inflammation reaction. The leukocytes within the tumor stroma, as well as the cytokines and growth factors derived from the leukocytes, construct the complex extrinsic pathway which plays a core role in the tumor formation process [10].

Tumor-associated macrophages (TAMs) are the principal leukocyte subset driving amplification of the inflammatory response in the tumor milieu. They primarily belong to the myeloid cell lineage and are derived from myeloid progenitor cells. These precursor cells are located in the bone marrow and upon maturation the monocytes are released into the bloodstream. Thereafter, certain chemokines, e.g. CCL2 and CCL5 recruits TAMs into the tumor stroma, where they facilitate the invasive neoplastic state.

TAMs assist in the malignant behavior of the tumor cell by releasing cytokines, growth factors and matrix-degrading enzymes and a host of angiogenic factors. Lists of molecular alterations are involved in the TAM-PC connection. For example, administration of agonist CD40 antibody activates macrophages that in turn infiltrate tumor turning it into tumoricidal, and facilitate the depletion of tumor stroma [11]. TAMs may also secrete MIP3 $\alpha$ to increase the migration ability of PC cells by binding to the transmembrane receptor CCR6 [12]

A study showed that TAMs could convey pro-angiogenetic effects to PC cells. Blocking the TIE2 ligand and angiogenic factor, angiopoietin-2, could impede the up regulation of TIE2 in TAMs and decrease tumor angiogenesis of PC [13]. Furthermore, TAMs help sustain the cancer stem cells, due to its chemo resistance, metastatic dissemination and the induction of immune suppression [14].

\subsection{Demographic Data}

From January to December 2014, 300 patients were treated at pancreatic center of Wuhan Union hospital in china. Out of 300 cases, 266 cases were PC and 34 were CP and the sex ratio in $\mathrm{PC}$ group was $1.98: 1$ and in $\mathrm{CP}$ group was 1.61:1according to table 1 data, thus showing a male preponderance in both diseases compared to female $(\mathrm{P}=0.001$,table 1$)$. $\mathrm{PC}$ patients ranged from 14 to 84 years with a mean of 59 years and $\mathrm{CP}$ patients ranged from 24 to 68 years with a mean of 46 years. This shows that PC patients are $10-15$ years older than $\mathrm{CP}$ patients. The statistical analysis was significant with a $\mathrm{P}$ value of 0.001 (less than 0.05). The average diameter of the lesions was 3.6 \pm 3.2 in PC group and $3.8 \pm 3.3$ in $\mathrm{CP}$ group as shown in table 1 and the most common lesions site was head of pancreas in both diseases. [1, 15]

Epidemiological report showed a parallel increase in both diseases in many countries. Niderau et al [16] found the prevalence of pancreatic cancer with $\mathrm{CP}$ to be $3.5 \%$ and Lowenfel et al [17] showed a prevalence of $2.9 \%$. This was in line with other calculations from 16 different studies published between 1960 and 1989 with an overall incidence of PC patients with $\mathrm{CP}$ to be $3 \%$ with a range from $0.8 \%$ to $3 \%$ ) which is higher than expected, therefore showing some interrelationship between CP and PC $[17,19]$.
Table 1. Demographic data and clinical presentation of pancreatic lesion in $P C$ and $C P$.

\begin{tabular}{llll}
\hline Parameters & $\begin{array}{l}\text { Pancreatic } \\
\text { cancer } \\
\text { N=266 }\end{array}$ & $\begin{array}{l}\text { Chronic } \\
\text { pancreatitis } \\
\mathbf{N = 3 4}\end{array}$ & P value \\
\hline $\begin{array}{l}\text { Mea nage (range); years } \\
\text { Gender }\end{array}$ & $59(14-84)$ & $46(24-680$ & 0.001 \\
Male & 174 & 22 & 0.001 \\
Femele & 92 & 12 & \\
Meanlesionsize(range);cm & $3.6+/-3.2$ & $3.8+/-3.3$ & 0.002 \\
ClinicalPresentation & 141 & 20 & \\
Diabetes & $141(53 \%)$ & $20(58.82 \%)$ & \\
Abdominal pain & $206(77.44 \%)$ & $25(73.52 \%)$ & 0.001 \\
Weightloss & $199(74.81 \%)$ & $21(67.76 \%)$ & \\
jaundice & $97(36.46 \%)$ & $11(32.03 \%)$ & \\
Antecedents & & & \\
Ulcer & $112(42.10 \%)$ & $14(41.17 \%)$ & \\
Cholecystectomy & $142(53.38 \%)$ & $17(50 \%)$ & 0.001 \\
Smoking & $180(67.66 \%)$ & $22(64.70 \%)$ & \\
Alcoholdrinking & $162(60.90 \%)$ & $21(61.76 \%)$ & \\
\hline
\end{tabular}

This table shows that PC patients are $10-15$ years older than CP patients which correspond to the time needed for the inflammation to propagate into cancerous cells. The statistical analysis was significant with a $\mathrm{P}$ value of 0.001 (less than 0.05). The average diameter of the lesions was 3.6 \pm 3.2 in $\mathrm{PC}$ group and $3.8 \pm 3.3$ in $\mathrm{CP}$ group $(\mathrm{P}=0.002)$.

The symptoms were found to be more common in $\mathrm{PC}$ patients with $77.4 \%$ cases of abdominal pain, $74.8 \%$ cases of weight loss and $36.5 \%$ cases of jaundice compared to $73.5 \%$, $61.8 \%$ and $32.4 \%$ cases of CP patients with abdominal pain, weight loss and jaundice respectively $(\mathrm{P}=0.001)$. The data was of no significance in relation to the clinical presentation of both diseases.

In both PC and CP groups' almost same percentage of patients were observed to have hyperglycemia as a direct consequence of diabetes mellitus $(\mathrm{P}=0.001)$.

\subsection{Clinical Symptoms}

According to table 1, the symptoms were found to be more common in PC patients with $77.4 \%$ cases of abdominal pain, $74.8 \%$ cases of weight loss and $36.5 \%$ cases of jaundice compared to $73.5 \%, 61.8 \%$ and $32.4 \%$ cases of CP patients with abdominal pain, weight loss and jaundice respectively. The data was of no significance in relation to the clinical presentation of both diseases which was in line with the literature of Ardengh et al [20,21].

From history, ulcer, cholecystectomy, smoking and alcohol drinking were found to be a antecedents of the pancreatitis disease. Person who smoked had a highter percentage in both $\mathrm{CP}$ and $\mathrm{PC}$ as shown in the table $1(\mathrm{P}=0.001$ less than 0.05$)$. Our results werere superimposable with majority of the meddicaleel literature [22].

Furthermore through this study, CA19-9, CEA, CYFRA21-1 and Ferritin are found to be the markers of both pancreatic cancer and chronic pancreatitis. However, other markers were also identified in different parts (table2).

Our study along with the image proves that $\mathrm{PC}$ and $\mathrm{CP}$ are 
more frequent in the body and tail of pancreas. (Table3) and (Figures A, B and C, D).

From history, ulcer, cholecystectomy, smoking and alcohol drinking were found to be a antecedents of the pancreatitis diseases.Person who smoked had a higher percentage in both $\mathrm{CP}$ and $\mathrm{PC}$ as shown in the table1. Our results were superimposable with majority of the medical literature [22].

Table 2. Furthermore through this study, CA19-9, CEA, CYFRA21-1 and Ferritin are found to be the markers of both pancreatic cancer and chronic pancreatitis. However, other markers were also identified in different parts.

\begin{tabular}{lll}
\hline Level of lesions & $\begin{array}{l}\text { Pancreatic cancer } \\
\text { N=266 }\end{array}$ & Chronicpancreatitis N=34 \\
\hline Head & $39(14.66 \%)$ & $4(11.76 \%)$ \\
Head-neck & $5(1.87 \%)$ & $2(5.88 \%)$ \\
Neck & $6(2.25 \%)$ & $4(11.76)$ \\
Body & $122(45.86 \%)$ & $14(41.17 \%)$ \\
Tail & $94(35.33 \%)$ & $12(35.29 \%)$ \\
\hline
\end{tabular}

Table 3. Our study along with the image proves that $P C$ and $C P$ are more frequent in the body and tail of pancreas.

\begin{tabular}{lll}
\hline Tumor marker & PC & CP \\
\hline CA19-9 & $138(51.87 \%)$ & $17(50 \%)$ \\
CA125 & $74(27.81 \%)$ & $8(23.52 \%)$ \\
CEA & $63(23.68 \%)$ & $8(23.52 \%)$ \\
Ferritin & $139(52.25)$ & $18(52.94 \%)$ \\
CA15-3 & $9(3.38 \%)$ & $6(17.64 \%)$ \\
CA72-4 & $24(9.02 \%)$ & $15(44.11 \%)$ \\
AFP & $5(1.87 \%)$ & $6(17.64 \%)$ \\
CYFRA21-1 & $55(20.67 \%)$ & $9(26.47 \%)$ \\
NSE & $60(22.55 \%)$ & $12(35.29 \%)$ \\
PSA & $2(0.75 \%)$ & $0(0.00 \%)$ \\
SCC & $20(7.51 \%)$ & $5(14.70 \%)$ \\
\hline
\end{tabular}

\subsection{Diabetes Mellitus}

In both PC and CP groups' almost same percentage of patients were observed to have hyperglycemia as a direct consequence of diabetes mellitus (table 1). This is a common observation made by several publications in china [23]America [24]andEurope [25].

Normally pancreas is a mixed gland of exocrine and endocrine. Exocrine consist of acinar cell and duct cell secretions whereas endocrine pancreas are made up of Islets of Langerhans which contain beta-cells (one of the many) that secretes insulin.

Due to pancreatic lesions in exocrine part, acinar tissue loss and replacement by fibrosis occurs. Moreover, Islets become smaller than normal and may be isolated from their vascular surrounding due to fibrosis. Eventually endocrine insufficiency (diabetes mellitus) occurs with progressive destruction of gland.

\subsection{Amino Transaminase}

Alkaline phosphatase (ALP) is an enzyme found in large amount in bone liver and bile duct tissue. Normally, level of ALP in the blood, range from 44 to $147 \mathrm{IU} / \mathrm{L}$. ALP has been showed to be present in pancreatic duct epithelium, acini, islets and tumors.

Gamma glutamyl Transferase (GGT) is an enzyme found in cell membranes of many tissues mainly in the liver, kidney and pancreas. The reference range is $0-51 \mathrm{IU} / \mathrm{L}$. GGT is often measured in conjunction with ALP to determine whether ALP increase is due to liver or pancreatic disease [23].

In our center, an increase in ALP and GGT in PC and CP groups can be observed in figure 1A and 1B. High level of both ALP and GGT can be potential marker of pancreatic disease, especially in those patients with suspected for chronic pancreatitis or pancreatic cancer [26, 27].
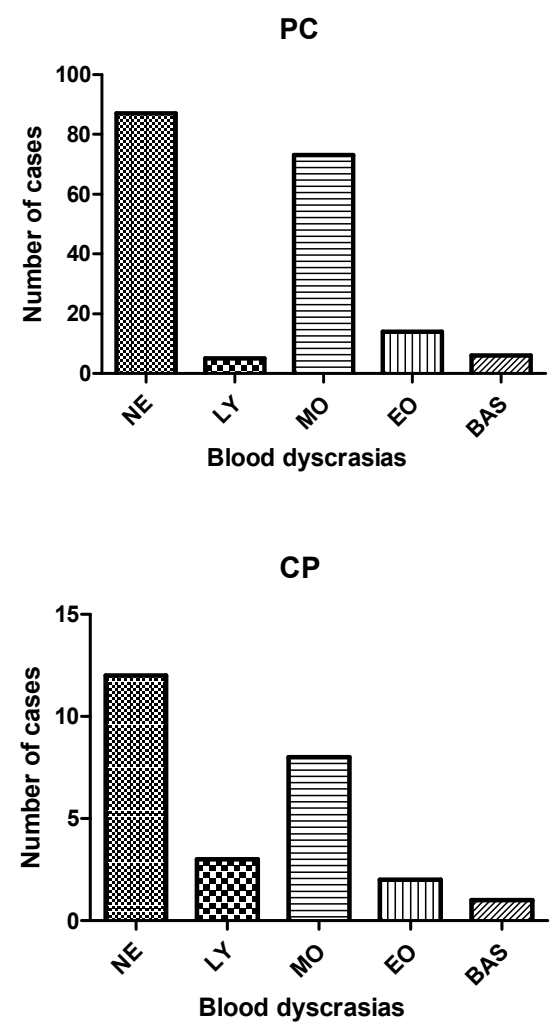

Figure $1 A$ and $1 B$. Shows the leukocyte levels compared to the normal range in PC and CP. NE (neutrophils), LY (lymphocytes), MO (monocytes), EO (eosinophils), BAS (basophils).

\section{Conclusion}

In summary, the etiologic factors that link $\mathrm{PC}$ and $\mathrm{CP}$ are as described: (1) the interactions of stromal -tumor result in a chaotic environment accompanied by loss of immune surveillance and repair response thereby developing a neoplastic events. (2) The peak age of PC patients has been observed to be 10-15 years older than CP, (table 1). (3) The lesion diameter was approximately the same in both diseases and the lesion site which was body and tail of pancreas was common (figures $2 \mathrm{~A}, 2 \mathrm{~B}, 2 \mathrm{C}, 2 \mathrm{D}$ ). (4) The body and tail of pancreas as a location has been showed by many publications $[1,13]$. (5) The incidence of both diseases increased simultaneously in many countries [29, 32]. (6) Man was more affected in both groups than female with a sex ratio of 
nearly $2: 1(\mathrm{p}=0.001$, table 1$)$.Clinical symptoms were of no great value since they hardly differ in $\mathrm{PC}$ and $\mathrm{CP}$. (7) Pancreatic insufficiency is a common factor in $\mathrm{PC}$ and $\mathrm{CP}$, thus diabetes Mellitus is witnessed equally. (8) ALP and GGT are the enzymes that revealed to act as potential markers for pancreatic lesions. (9) Neutrophiles, lymphocytes were high in the pancreatic lesions patients but higher in chronic pancreatic patients than pancreatic canceras shown in figure $1 \mathrm{~A}$ and $1 \mathrm{~B}$ along with figure $3 \mathrm{~A}$ and $3 \mathrm{~B}$. All these etiologic link factors and their respective results may act as
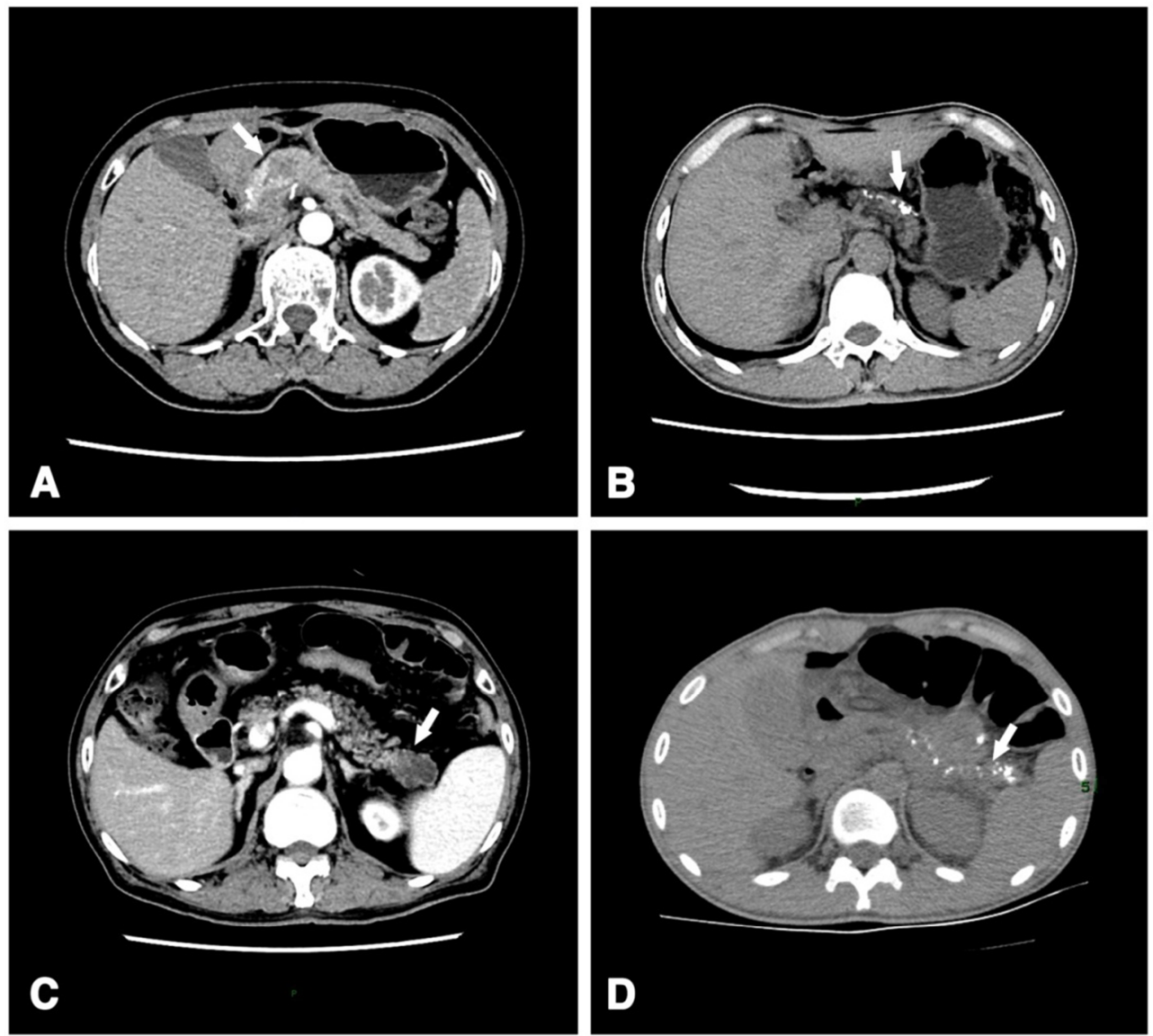

Figure $2 A, 2 B$, and $2 C, 2 D$. Show us, the most frequent localizations in the pancreatic cancer, and chronic pancreatitis. According to the results the body and the tail of the pancreas are the preferential sites $P C$ and $C P$.
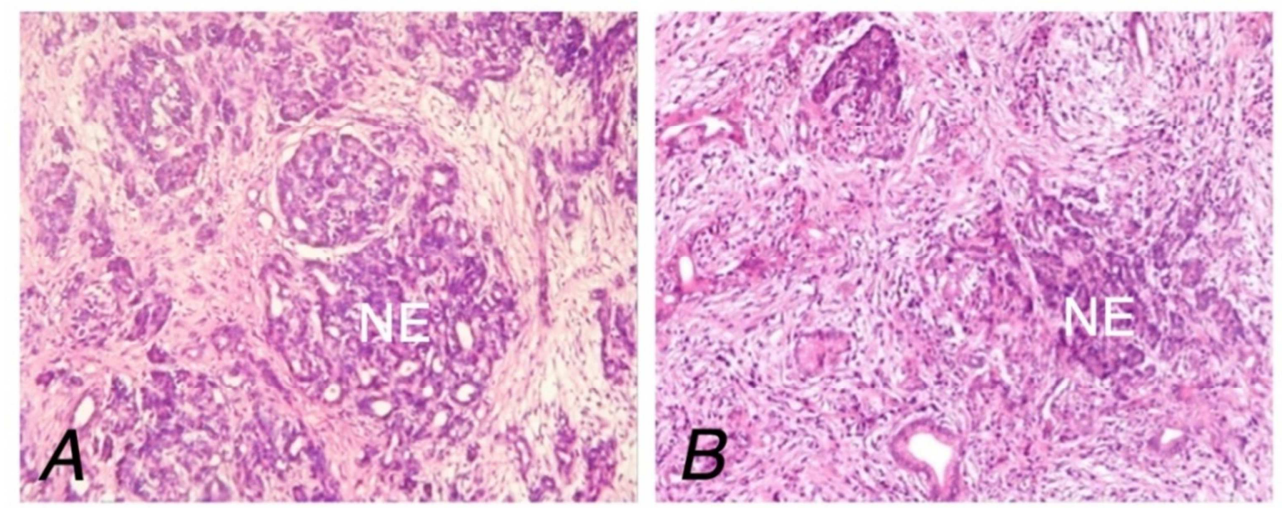

Figure $3 A(C P)$ and $3 B(P C)$. Provide a histopathological evidence to compare the high level of neutrophils in PC and CP. The neutrophiles are marked with $(N E)$. 


\section{Acknowledgement}

We would like to thank the professor Chunyou Wang, head of teacher, Director of Pancreatic disease institute of Wuhan union hospital for his support and critical reading of the manuscript.

\section{Conflict of Interest Statement}

The authors declare that there is no conflict of interest with any financial organization or corporation or individual that can inappropriately influence this work.

\section{References}

[1] Camara, S. N., Ramdany, S., Balde, O. T., Barry, A. B., \& Adji, S. (2015). Etiology, pathology, management and prognosis of chronic pancreatitis in Chinese population: A retrospective study. J Huazhong UnivSci Technolog MedSci, $35,384-389$.

[2] Jemal, A., Siegel, R., Xu, J., \& Ward, E. Cancer statistics. $C A$ cancer JClin 2010, 60, 277-300.

[3] Koninger, J., Friess, H., Muller, M., \& Buchler, M. W. (2004). Duodenum preserving pancreatic head resection in the treatment of chronic pancreatitis. Rocz Akad Med Bialymst, 49, 53-60.

[4] Balkwill, F., \& Mantovani A. Inflammation and cancer: back to Virchow? The lancet, 2001, 357, 539-545.

[5] Pinho, A. V., Chantrill, L., \& Rooman, I. (2014). Chronic pancreatitis: a path to pancreatic cancer. Cancer letters, 345(2), 203-209.

[6] Tuveson, D. A., \& Neoptolemos, J. P. (2012). Understanding metastasis in pancreatic cancer: a call for new clinical approaches. Cell, 148, 21-23.

[7] MitchellP. Fink. Partiallist of physiologic effects induced by infusing interleukin 1 or tumor necrosis factor into human subjects. Sabiston textbook of surgery $19 \mathrm{ed}$ page 48 .

[8] Raimondi, S., Lowenfels, A. B., Morselli-Labate, A. M., Maisonneuve, P., \& Pezzilli, R. Pancreatic cancer in chronic pancreatitis; aetiology, incidence, and early detection. Best practice \& research Clingastroenterolog, 2010, 24, 349-358.

[9] Szasz, G. (1969). A kinetic photometric method for serum $\gamma$ glutamyltranspeptidase. Clinchemist, 15, 124-136.

[10] Lum, G., \& Gambino, S. R. Serum gammaglutamyltranspeptidase activity as an indicator of disease of liver, pancreas, or bone. Clin Chemist, 1972, 18, 358-362.

[11] Beatty, G. L., Chiorean, E. G., Fishman, M. P., Saboury, B., Teitelbaum, U. R., et al. CD40 agonists alter tumor stroma and show efficacy against pancreatic carcinoma in mice and humans. Sci, 2011, 331, 1612-1616.

[12] Bedi, M. M. S., Gandhi, M. D., Jacob, G., Lekha, V., Venugopal, A., et al.. CA 19-9 to differentiate benign and malignant masses in chronic pancreatitis: is there any benefit? $I J G, 2009,28,24-27$.
[13] Campbell, A. S., Albo, D., Kimsey, T. F., White, S. L., \& Wang, T. N. Macrophage inflammatory protein-3 $\alpha$ promotes pancreatic cancer cell invasion Surg Res, 2005, 123, 96-101.

[14] Mazzieri, R., Pucci, F., Moi, D., Zonari, E., Ranghetti, A., et al. Targeting the ANG2/TIE2 axis inhibits tumor growth and metastasis by impairing angiogenesis and disabling rebounds of proangiogenic myeloid cells. Cancer cell, 2011, 19, 512-526.

[15] Mitchem, J. B., Brennan, D. J., Knolhoff, B. L., Belt, B. A., Zhu, Y., et al. (2013). Targeting tumor-infiltrating macrophages decreases tumor-initiating cells, relieves immunosuppression, and improves chemotherapeutic responses. Cancer research, 2013, 73, 1128-1141.

[16] Kaiser, M. H., \& Ellenberg, S. S. (1985). Pancreatic cancer: adjuvant combined radiation and chemotherapy following curative resection. Arch Surg, 1985, 120, 899-903.

[17] Niederau, C., \& Grendell, J. H. (1992). Diagnosis of pancreatic carcinoma: imaging techniques and tumor markers. Pancreas, 7(1), 66-86.

[18] Yeo, T. P., \& Lowenfels, A. B. (2012). Demographics and epidemiology of pancreatic cancer. The CA J, 2012, 18, 477-484.

[19] Bosetti, C., Bertuccio, P., Negri, E., La Vecchia, C., Zeegers, M. P., et al. Pancreatic cancer: overview of descriptive epidemiology. MolCarc, 2012, 51(1), 3-13.

[20] Lowenfels, A. B., Walker, A. M., Althaus, D. P., Townsend, G., \& Domellöf, L. Gallstone growth, size, and risk of gallbladder cancer: an interracial study. Int J Epidemiol 1989, $18,50-54$.

[21] Andren-Sandberg, A., Dervenis, C., \& Lowenfels, B. Etiologic links between chronic pancreatitis and pancreatic cancer. Scan J G, 1997, 32, 97-103

[22] Albert B. Lowenfels, Patrick. Maisonneuve, Giorgio Cavallini, Rudolf W. Ammann, Paul G.Lankish, Jens R. Andersen, Eugene P.Dimanno, Ake .Andren-sandberg, Lennart Domellof and the international pancreatitis study group; pancreatitis and the risk of pancreatic cancer. The new England journal of Medecine May 20.1993. Volume 328. Number 20.

[23] Ardengh, J. C., Lopes, C. V., Campos, A. D., Pereira de Lima, L. F., Venco, F., et al. Endoscopic ultrasound and fine needle aspiration in chronic pancreatitis: differential diagnosis between pseudotumoral masses and pancreatic cancer. JOP, 2007, 8, 413-421.

[24] Rickels, M. R., Bellin, M., Toledo, F. G., Robertson, R. P., Andersen, D. K., et al. (2013). Detection, evaluation and treatment of diabetes mellitus in chronic pancreatitis: recommendations from PancreasFest 2012. Pancreatol, 2013 $13,336-342$.

[25] Zhang, C., Yang, G., Ling, Y., Chen, G., \& Zhou, T. (2014). The early diagnosis of pancreatic cancer and diabetes: what's the relationship? J GastrointestOncol, 2014, 5, 481-488.

[26] Falconi, M., Casetti, L., Salvia, R., Sartori, N., Bettini, R., et al. (2000). Pancreatic head mass, how can we treat it? Chronic pancreatitis: surgical treatment. JOP2000, 1, 154-161.

[27] Carr-Locke, D. L., \& Davies, T. J. Pancreatic juice gammaglutamyltransferase, alanine transaminase, and alkaline phosphatase in pancreatic disease. Digest Dis Sci, 1980, 25, 374-378. 
[28] Wilson Denise, D. Manual of Laboratory \& Diagnostic Tests. 2008.

[29] Lévy, P., Dominguez Munoz, E., Imrie, C., Löhr, M., \& Maisonneuve, P. (2014). Epidemiology of chronic pancreatitis: burden of the disease and consequences. United European gastroenterology journal, 2(5), 345-354.

[30] Smailyte, G., Jasilionis, D., Vincerzevskiene, I., Krilaviciute, A., Ambrozaitiene, D., Stankuniene, V., \& Shkolnikov, V. M. (2015). Educational differences in incidence of cancer in Lithuania, 2001-2009: evidence from census-linked cancer registry data. European Journal of Cancer Prevention, 24(3), 261-266.

[31] Siegel, R., Ma, J., Zou, Z., \&Jemal, A. (2014). Cancer statistics, 2014. CA: a cancer journal for clinicians, 64 (1), 929.

[32] Lennon, A. M., Wolfgang, C. L., Canto, M. I., Klein, A. P., Herman, J. M., Goggins, M., \&Hruban, R. H. (2014). The early detection of pancreatic cancer: what will it take to diagnose and treat curable pancreatic neoplasia?.Cancer research, 74(13), 3381-3389. 